\title{
Anticancer drugs and COVID-19 antiviral treatments in cancer patients: what can we safely use?
}

\author{
Paul Gougis ${ }^{1}$, Charlotte Fenioux ${ }^{1}$, Christian Funck-Brentano ${ }^{2}$, Marianne Veyri ${ }^{3}$, Joseph \\ Gligorov $^{4}$, Caroline Solas ${ }^{5}$, and Jean-Philippe Spano ${ }^{3}$ \\ ${ }^{1}$ Hopital Pitie-Salpetriere Service de Pharmacologie \\ ${ }^{2}$ Affiliation not available \\ ${ }^{3}$ Pitié Salpêtrière University Hospital Department of Oncology Radiotherapy \\ ${ }^{4}$ Sorbonne Universite \\ ${ }^{5}$ AP-HM, Hôpital La Timone
}

April 29, 2020

To the editor,

The rapid emergence of COVID-19 pandemics worldwide is of particular concern for fragile populations who are more at risk of acute respiratory distress syndrome and death. Patients treated for malignant hemopathy and solid cancers have a four times higher risk of hospitalization due to influenza infection, and a ten times higher risk of death. This fragility could be due to their age, multiple associated comorbidities, lymphopenia, or the immunosuppressive action of a broad spectrum of anticancer drugs.[1] Therefore, anticancer drugs should be used carefully in this population. Without further safety data, it might be unsafe to treat SARSCoV-2 positive patients who have COVID-19 symptoms with anticancer drugs known to increase infections or harvesting immunosuppressive properties. We summarized in the table (part A) drug classes that have been reported to increase either neutropenia or infections. Regarding patients tested positive who have recovered from their symptoms, clinical data is missing. It is currently not clear if cancer treatments should be stopped, and if so, the time needed to resume it safely.

In any of these settings, clinical trials and incoming standard of care could lead to the prescription of antiviral drugs concomitant to non-immunosuppressive anticancer treatments. Similar to previous works reporting interactions between HIV antiretrovirals and anticancer drugs, [2] these two classes of medications have a narrow therapeutic index and can have pharmacological interactions. Some of them are substrates or interact with hepatic cytochrome P450 cytochrome isoenzymes (CYP), particularly CYP3A4, and pharmacokinetic interactions could lead to supra or infratherapeutic concentrations. For example, enzalutamide, a nonsteroidal antiandrogen prescribed for prostate cancer, is both a CYP3A4 substrate and inducer. Ritonavir, on the other hand, is a pharmacokinetic booster of lopinavir contained in Kaletra, which is explored as a COVID-19 treatment. Ritonavir is a substrate and also a potent inhibitor of CYP3A4. Thus, enzalutamide and ritonavir could interfere with each other's metabolism, decrease or increase each other's clearance, and be responsible for severe toxicities or decreased efficacy. Favipiravir, an anti-EBOV drug, also a candidate for the COVID-19 treatment, is an inhibitor of CYP2C8,[3] and therefore may increase anticancer drug metabolized through this pathway, such as dabrafenib and enzalutamide. Furthermore, CYP3A4 induction could lead to sustained CYP3A4 increased activity for up to 1 week after discontinuation. Dabrafenib or enzalutamide, two CYP3A4 inducers, could significantly decrease hydroxychloroquine concentration during the first week of wash-out. 
Among pharmacodynamic interactions, QT interval prolongation could be of particular interest. Hydroxychloroquine, which is currently widely prescribed as an anti-coronavirus drug, or azithromycin, are two drugs known to prolong QT interval. Concomitant use of QT-prolonging anticancer drugs could lead to Torsadede-Pointes and be fatal. Caution should be observed in this case, and electrocardiographic monitoring should be implemented to monitor QT interval duration during combination therapy.

Similarly, anticancer drugs could potentiate nephrotoxicity and hepatotoxicity of antiviral treatments.

The table (part B) summarizes pharmacokinetic and pharmacodynamics interactions between some currently tested drugs against COVID-19 and anticancer drugs.

Table:

\section{A - Class of anticancer drugs with immunosuppressive properties}

Immunosuppressing drugs were defined as drugs associated with significantly more infections or neutropenia compared to the control group or placebo in trials. These drugs were excluded from part B.

B - Summary of pharmacokinetic (PK) and pharmacodynamic (PD) interactions of interest concerning non-immunosuppressive anticancer drugs and potential COVID-19 treatments.

No interaction driven by other cytochromes were found. Red arrows are for interactions relying on clinically significant data. Orange arrows are for interactions relying on in vitro data for pharmacokinetic interactions. Cytochromes involved in the drug interaction were specified. Substrates for which induction but not inhibition could lead to significant interaction are between brackets. When the interaction modifies the pharmacokinetics of the anticancer drug, the arrow was on the bottom-left. Antiviral exposition prediction is on the top-right. Red boxes are for anticancer drugs with known Torsade-de-Pointes risk and high risk of renal and liver toxicities. Orange boxes are for anticancer drugs prolonging QT without known Torsade-de-Pointes risk and moderate risk for renal and liver toxicities.

Data from FDA labels [4] were retrieved for drug metabolism, QT prolongation, and nephrotoxicity. Livertox database was used for hepatotoxicity[5].

A-

\begin{tabular}{llll}
\hline Immunosuppressing classes of drugs & Cytotoxic chemotherapy & Proteasome inh. & Histone deacetylase inh \\
\hline & BCR-ABLi & FLT3i & MEKi \\
& CDK4-6i & PARPi & Multikinase inh. (sorafe \\
\hline
\end{tabular}

B-

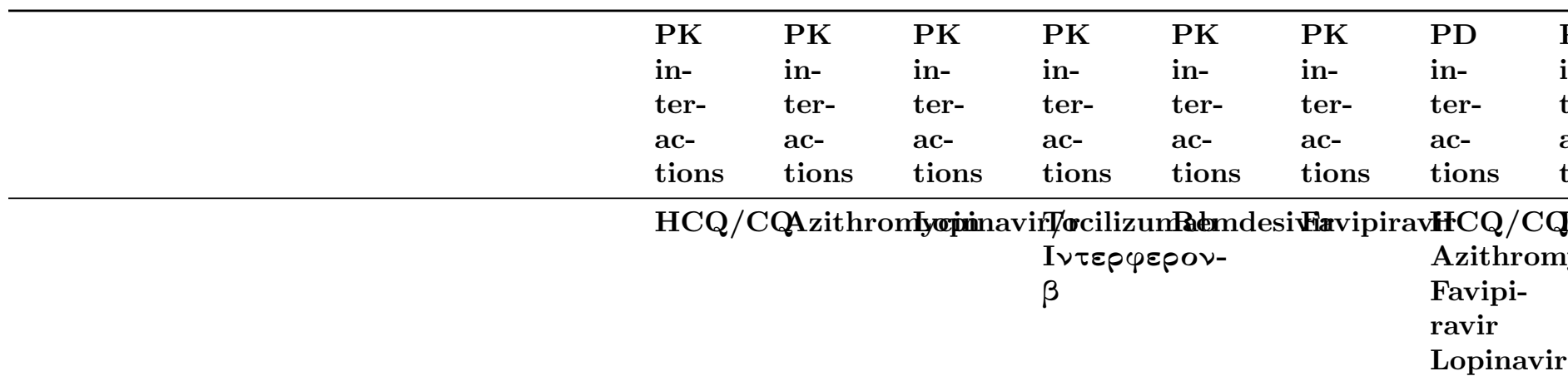




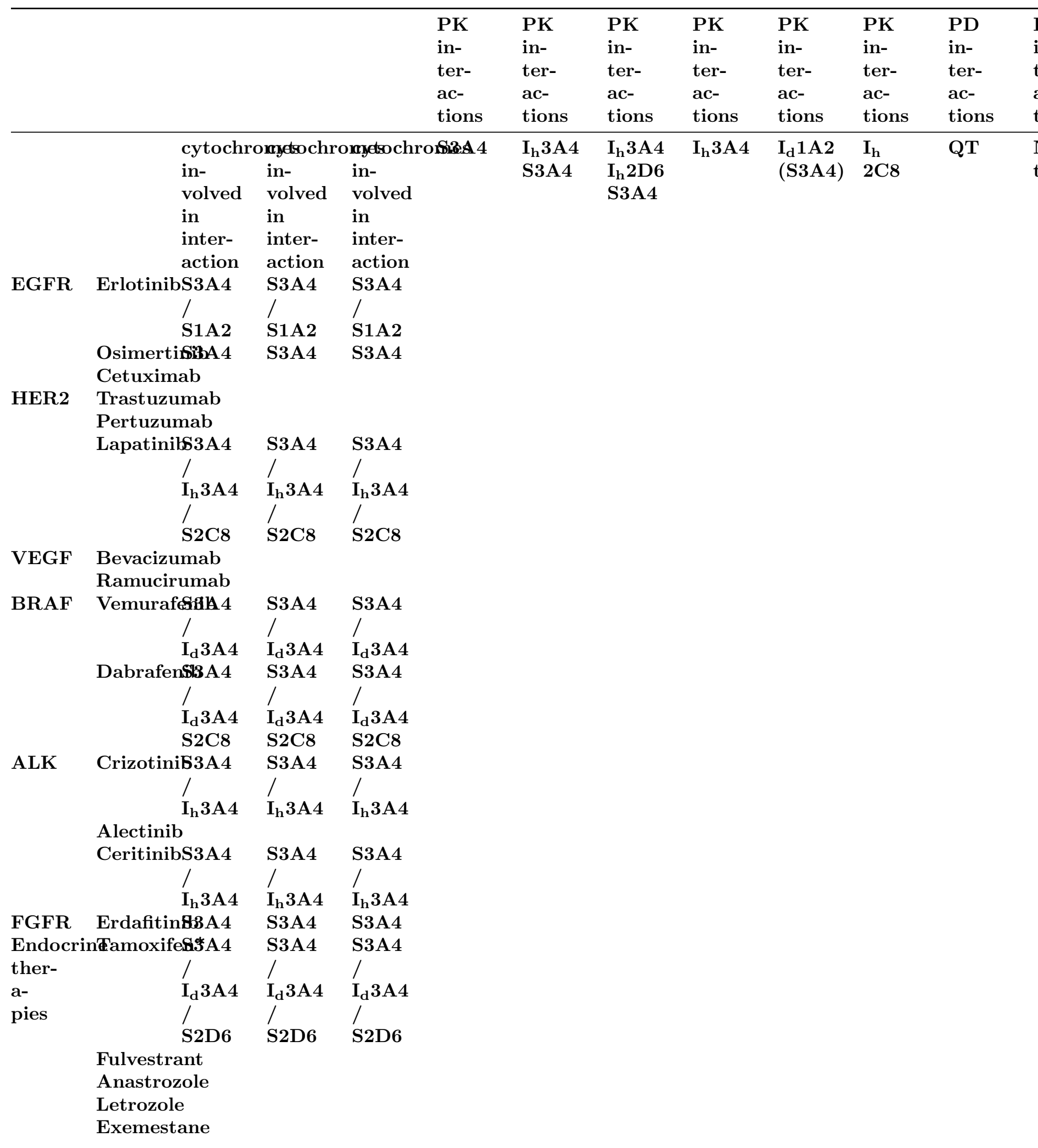




\begin{tabular}{|c|c|c|c|c|c|c|c|c|c|}
\hline & & & $\begin{array}{l}\text { PK } \\
\text { in- } \\
\text { ter- } \\
\text { ac- } \\
\text { tions }\end{array}$ & $\begin{array}{l}\text { PK } \\
\text { in- } \\
\text { ter- } \\
\text { ac- } \\
\text { tions }\end{array}$ & $\begin{array}{l}\text { PK } \\
\text { in- } \\
\text { ter- } \\
\text { ac- } \\
\text { tions }\end{array}$ & $\begin{array}{l}\text { PK } \\
\text { in- } \\
\text { ter- } \\
\text { ac- } \\
\text { tions }\end{array}$ & $\begin{array}{l}\text { PK } \\
\text { in- } \\
\text { ter- } \\
\text { ac- } \\
\text { tions }\end{array}$ & $\begin{array}{l}\text { PK } \\
\text { in- } \\
\text { ter- } \\
\text { ac- } \\
\text { tions }\end{array}$ & $\begin{array}{l}\text { PD } \\
\text { in- } \\
\text { ter- } \\
\text { ac- } \\
\text { tions }\end{array}$ \\
\hline 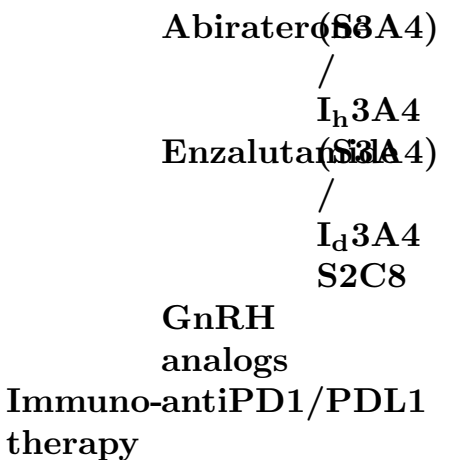 & $\begin{array}{l}(\mathrm{S} 3 \mathrm{~A} 4) \\
/ \\
\mathrm{I}_{\mathrm{h}} 3 \mathrm{~A} 4 \\
(\mathrm{~S} 3 \mathrm{~A} 4) \\
/ \\
\mathrm{I}_{\mathrm{d}} 3 \mathrm{~A} 4 \\
\mathrm{~S} 2 \mathrm{C} 8\end{array}$ & $\begin{array}{l}(\mathrm{S} 3 \mathrm{~A} 4) \\
/ \\
\mathrm{I}_{\mathrm{h}} 3 \mathrm{~A} 4 \\
(\mathrm{~S} 3 \mathrm{~A} 4) \\
/ \\
\mathrm{I}_{\mathrm{d}} 3 \mathrm{A4} 4 \\
\mathrm{~S} 2 \mathrm{C} 8\end{array}$ & & & & & & & \\
\hline
\end{tabular}

*Tamoxifen is a prodrug and the reported effect is on the active metabolite endoxifen

CQ: chloroquine; GnRH: gonadotrophin-releasing hormone; HCQ: hydroxychloroquine; $I_{d}$ : cytochrome inducer; $I_{h}$ : cytochrome inhibitor; Lopinavir/r: lopinavir/ritonavir association (KALETRA ß); S: substrate

\section{Conflict of interest statement}

JPS: consultant: Roche, MSD, Biogaran ; adboard/symposium: MSD, Roche, AZ, Leopharma, Mylan, Pfizer, BMS, Novartis, PFO, Myriads, Gilead, Lilly. All remaining authors have declared no conflicts of interest.

\section{References}

1. Liang W, Guan W, Chen R et al. Cancer patients in SARS-CoV-2 infection: a nationwide analysis in China. Lancet Oncol. 2020; 21(3):335-337.

2. Spano J-P, Poizot-Martin I, Costagliola D et al. Non-AIDS-related malignancies: expert consensus review and practical applications from the multidisciplinary CANCERVIH Working Group. Ann. Oncol. Off. J. Eur. Soc. Med. Oncol. 2016; 27(3):397-408.

3. Liverpool COVID-19 Interactions. 2020 [https://www.covid19-druginteractions.org/].

4. Drugs@FDA: FDA-Approved Drugs. 2020 [https://www.accessdata.fda.gov/scripts/cder/daf/].

5. LiverTox: Clinical and Research Information on Drug-Induced Liver Injury, Bethesda (MD): National Institute of Diabetes and Digestive and Kidney Diseases, 2012. 